\title{
Accelerated Wound Healing by a Topical Wound Healing Lipogel in Patients Undergoing Catheter De-placement - Evidence from a Randomized, Controlled Clinical Study
}

\author{
Thomas Eberlein $^{1}$, Sami Siam ${ }^{2}$ \\ ${ }^{1}$ Department of Surgery, College of Medicine and Medical Science, Arabian Gulf University, Manama, Bahrain \\ ${ }^{2}$ Department of nephrology, University hospital Muenster, Westphalian Wilhelms University, Muenster, Germany
}

Email address:

thomaseberlein@hotmail.com (T. Eberlein)

\section{To cite this article:}

Thomas Eberlein, Sami Siam. Accelerated Wound Healing by a Topical Wound Healing Lipogel in Patients Undergoing Catheter Deplacement - Evidence from a Randomized, Controlled Clinical Study. Journal of Surgery.

Vol. 8, No. 2, 2020, pp. 48-55. doi: 10.11648/j.js.20200802.12

Received: January 31, 2020; Accepted: February 21, 2020; Published: March 17, 2020

\begin{abstract}
Objective: The purpose of this clinical study was to assess a novel wound model and to compare the wound healing properties of a topical wound healing hydroactive lipogel $\left(\mathrm{MediGel}^{\mathbb{B}}\right)$ promoting moist wound environment versus standard treatment by a standard plaster und dry environmental conditions in patients undergoing catheter de-placement as a model for non-acute wounds affecting all skin layers in real life conditions (moist environment does improve granulation \& epithelialization supported by Lipogel - the MEDIGEL trial). Methods: Patients $(\mathrm{n}=69)$ admitted to the dialysis ward of a tertiary care institution with acute or chronic renal insufficiency and in need for dialysis were enrolled into a prospective, observer-blind, randomized, controlled, inter-individual experimental comparison study. Patients enrolled were undergoing placement of Sheldon multi-lumen catheter (11 French diameter) in the external jugular vein at the neck by Seldinger technique to enable access for hemodialysis. One group of patients which had catheter displaced after one session of hemodialysis was regarded as acute wound group. A different group of patients had catheter withdrawn after $14(+/-2$ days) and was classified as non-acute wound group. Topical treatments were randomly allocated, i.e. traditional care with standard plaster (control group) or investigational product (promoting moist environment) beneath a standard plaster (investigational product). Wound healing was assessed (digital photography, visual scoring) and analyzed via comparison of area under curve at day 0,3 and 7 after displacement of catheter. Results: Evaluation showed significantly faster wound healing results for the investigational product in comparison to standard (all time points) and with significant AUC difference in both patient groups, acute and non-acute. Visible re-epithelialization was recorded from day 3 to day 7 in acute wounds, delayed in non-acute wounds. Standard plaster-treated wounds remained open and had markedly larger wound area. Formation of fibrous scar tissue was minimal but less prominent in patients treated with the hydroactive lipogel in the non-acute group due to faster epithelialization promoted by moist environment. Conclusion: Clinically relevant accelerated epithelialization and faster wound healing were observed for the investigational product compared to traditional treatment indicating superiority of moist wound environmental conditions both in acute and non-acute wounds after displacement of catheter placed into the jugular vein for 5 hours or 2 weeks in patients undergoing hemodialysis. The model is an innovative approach to study acute and nonacute wounds affecting all three skin layers and should be further investigated.
\end{abstract}

Keywords: Acute and Chronic Wound Healing, Novel Wound Model, Lipogel

\section{Introduction}

Skin lesions affecting all layers of the skin (epidermisdermis and basal membrane) can have multiple and various causes derived from heterogeneous etiology such as acute, e.g. accidental trauma, intentional incisions, or chronic mainly due to an underlying disease and imbalanced physiological environment needed for re-epithelization. Abrasive and minor everyday wounds are usually treated by the patients themselves, typically covered with first aid 
dressings or left unprotected. Likewise, post-interventional surgical incisions are usually covered by standard dressings and traditional plasters which generate a dry wound environment. However, latest recommendations and advanced modern approaches promote a moist wound healing environment in skin care as it accelerates epithelialization and facilitates wound healing [1-5].

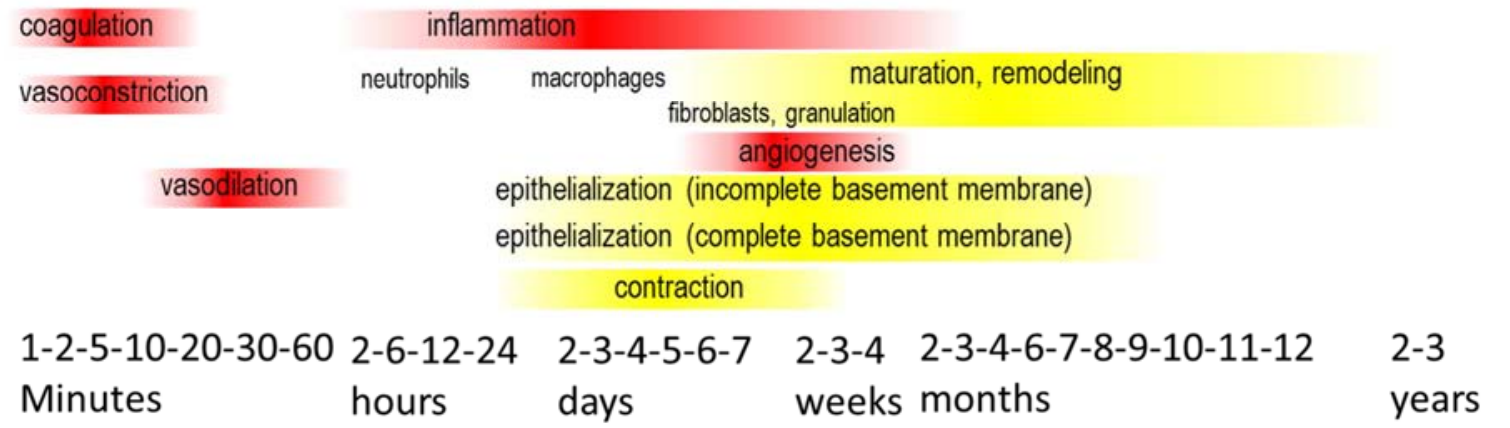

Figure 1. Wound types. Acute versus non-acute and chronic wounds follow several stages of healing (modified from Martin P, Nunan R (2015).

In line with these recommendations, the investigational product $^{1}$ applied is a hydroactive acidic lipogel which creates a moist wound environment and provides the $\mathrm{pH}$ value in an acidic range between 3.1-3.3. It contains trace elements (zinc and iron), nourishing oils and vitamin E [6]. This lipogel is a certified medical device class IIb as its main mechanism of action is physical in nature, providing a moist wound environment that helps to increase the rate of epithelialization $[7,8]$. The product is indicated for the treatment of acute wounds such as abrasions, cuts, scratches, lacerations, blistering burns, sunburns and cleansed, low exuding noninfected chronic wounds such as pressure ulcers $\mathrm{II}^{\circ}$ under supervision of professional medical staff [6].

In a porcine model with standardized induced skin lesions it has been shown that this product improves healing faster and re-epithelialization and closing of abrasive wounds was achieved in half the time in comparison to the wellestablished wound healing ointment ${ }^{2}$ [7] based on a paraffinwax/petrolatum/lanolin-complex based ointment containing dexpanthenol [6].

The hydroactive lipogel has also been investigated in a controlled study among healthy volunteers with induced abrasive skin lesions proving a faster rate of wound closure with hydroactive lipogel compared to standard plaster and less formation of crusts in comparison to established ointments [8].

The clinical evidence for hydroactive lipogel is based on established wound models [10-12]. Wound models are the most effective way to measure the treatment efficacy for acute wounds objectively in a clinical setting [11]. In human studies, they require the creation of small and possibly identical standardized wounds allowing comparing different treatments on an intra-individual basis in healthy volunteers. Established wound models apply tape stripping, suction by vacuum pumps, first-degree burns, mechanical abrasion using an emery wheel, an electrical dermatome or brush techniques with a sterile surgical hand brush. All these different methods resulting in dermal-epidermal defects allowing the measurement of re-epithelialization [10-12].

In contrast, we conducted an efficacy study under real-life clinical settings introducing a novel model with standardized incisions affecting all layers of the skin in patients with acute and non-acute wounds. "Acute" situation has been defined as an incision present for 4 to 6 hours; "non-acute" was defined as $14( \pm 2)$ days. This differentiation is of importance due to the fact that the process of wound healing follows several stages over time as depicted in Figure 1. We investigated the wound healing capacity of the investigational product in comparison to traditional treatment with standard plasters (control) in an observer blinded design.

\section{Methods}

\subsection{General Description}

For purpose of this study we recruited patients with acute or chronic renal insufficiency admitted to a tertiary care hospital in need for hemodialysis not via umbilical shunt but via an external multi-lumen catheter (Sheldon with 11 French in diameter) placed by modified Seldinger technique [13-14]. After skin disinfection of the neck with a standard disinfectant (octenidine phenoxyethanol), the right or left external jugular vein was punctured with a sharp hollow needle assisted by ultrasound guidance. A round-tipped guidewire was advanced through the lumen of the needle, the needle was withdrawn. A minor incision through all three layers of the skin was made using a stylet size 11 . Subsequently, a sheath was passed over the guidewire into the vein in order to enlarge the incision bluntly. After passing the multi-lumen Sheldon catheter, the guidewire was withdrawn (Figure 2).

1 MediGel®.

2 Bepanthen ${ }^{\circledR}$ Wund- und Heilsalbe. 


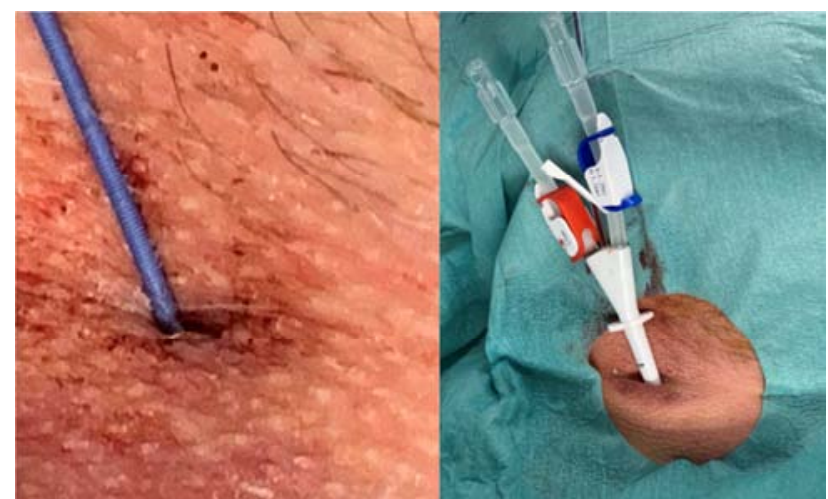

Figure 2. Representative images of a standardized incision into the jugular vein in one subject at baseline (day 0).

When the Sheldon catheter had been displaced, no sealing device was used to close the incision, but standard manual compression for 3 minutes was applied. After photo documentation of incision (baseline, day 0), patients were randomly assigned to have the incision covered solely by standard plaster or by an approx. $5 \mathrm{~mm}$ layer of investigational product covered by the identical type of standard plaster. Digital photos were also obtained at day 3 and 7. Visual scoring and measurements of wound area was performed by digital microscopy, a histological image analysis program for measuring wound areas (imageJ, National Institute of Health and University of Wisconsin) by an only one, blinded observer.

Patients were recruited from polyclinic emergency department or dialysis ward of a tertiary care center. The study was approved by the local Independent Ethics Committee (IEC) Northern Westphalia in Germany.

\subsection{Study Objectives}

The study had three major objectives, to investigate

1. A routine approach with standardized incisions set and utilized as acute or non-acute wound model

2. The healing properties and relative wound healing rate of a topical wound healing lipogel in comparison to traditional standard plaster as control (traditional treatment)

3. The outcome of acute and non-acute wounds treated with the different approaches

The use of standard plaster (dry environment) for wound care is not only a standard after catheter displacement, but also used in controlled trials investigating wound gels due to the unavailability of a practicable different approach, i.e. placebo is not feasible [15-17]. The investigation was a monocentric, observer-blinded, randomized, controlled, experimental study. The aim of the statistical analyses was the assessment of wound healing efficacy in skin wounds by the hydroactive lipogel in comparison to standard care in an inter-individual compare.

\subsection{Study Population, Inclusion and Exclusion Criteria}

Patients admitted to the dialysis ward were recruited into the study. Only subjects meeting all inclusion criteria were considered for the enrollment into the trial, i.e. men and women aged 18 to 75, skin type I to III (Fitzpatrick) [18] and in need for catheter placement were included after enlightenment and written consent. The main exclusion criteria were active skin disease, hairy skin or scars at the injured area, psoriasis and/or Lichen ruber and/or atopic dermatitis, history of keloids and hypertrophic scars, a history of plaster sensitivity or intake of drugs strongly interfering with the immune system (corticosteroids, immuno-suppressants) within up to 30 days before the start of the study as well as during the study period.

\subsection{Randomization \& Blinding}

The study was conducted under observer-blind conditions which was ensured by taking images of skin lesions at day 0,3 , and 7 which were analyzed by an observer without any informstion about the type of intervention. Due to the different application between the both groups, the patients and care givers involved in the application have not been blinded. All responsible persons for skin assessment, the visual and instrumental measurements, data management as well as trial statistics remained blinded during the complete conduct.

\subsection{Types of Wounds}

Seldinger technique applied [13, 14] leads to bloody skin incision affecting epidermis, dermis, and subcutis, adventitia tissue and puncture of jugular vein (Figure 2). If catheter was installed only for one session of hemodialysis, then catheter was displaced approx. 4 to 6 hours after initial incision. These incisions were classified as acute wounds where coagulation, vasocontraction, dilatation have taken place and inflammation (with the migration of neutrophils) has been initiated (Figure 1). If the catheter was placed for several dialysis sessions and kept for two weeks (14 \pm 2 days), the process of wound healing has advanced with progressed inflammation, migration of macrophages, granulation formation and remodeling initiated by fibroblasts. These incisions were classified as nonacute (Figure 1). Thus, our patient population has been splitted into two groups representing the acute versus the non-acute phase of wound healing (Figure 3).

\subsection{Study Product Application \& Treatments}

The investigational product as well as the standard plaster were applied topically appropriate to the instruction for use from day 0 till day 7 after documentation and visual assessment. A at least $5 \mathrm{~mm}$ thick layer of the investigational product was applied to the wound (after manual compression), extending well beyond the wound's margins and covered with standard plaster at day 0,3 and 5. The standard plasters applicated were selfadhesive, CE marked class I medical devices ${ }^{3}$.

During the first two days the subjects were not allowed to wetting the dressing. During the whole study period, subjects were not allowed to take a bath, to go swimming or to sauna, to conduct sporting or exhausting activities, to use cosmetics/moisturizers or

3 Cutiplast steril, $66001478,7.2 \mathrm{~cm}$ x 5cm, Smith Nephew. 
to wash the injured areas by using detergents.

\subsection{Assessments: Wound Healing (Quantitative Image Analysis \& Visual Assessment), Safety and Local Effects}

\subsubsection{Wound Healing}

At days 0,3 , and 7, digital photographs of the wounds were taken by the same trained technician. Image acquisition was performed with a handheld camera and a tubular distance holder (complete extinction of light) to enable reproducible images with defined magnification. The captured area was $2 \mathrm{~cm}$ in diameter. Pictures were taken before applying investigational gel or plaster. Afterwards the open wound area was marked by a trained and blinded observer on the pictures and its size calculated by the analyzing software. Comparing baseline wound margin (day 0 ) and follow up (day 3 and 7) allowed the calculation of the ratio at baseline (day 0 ) and current situation (Figure 3 ).

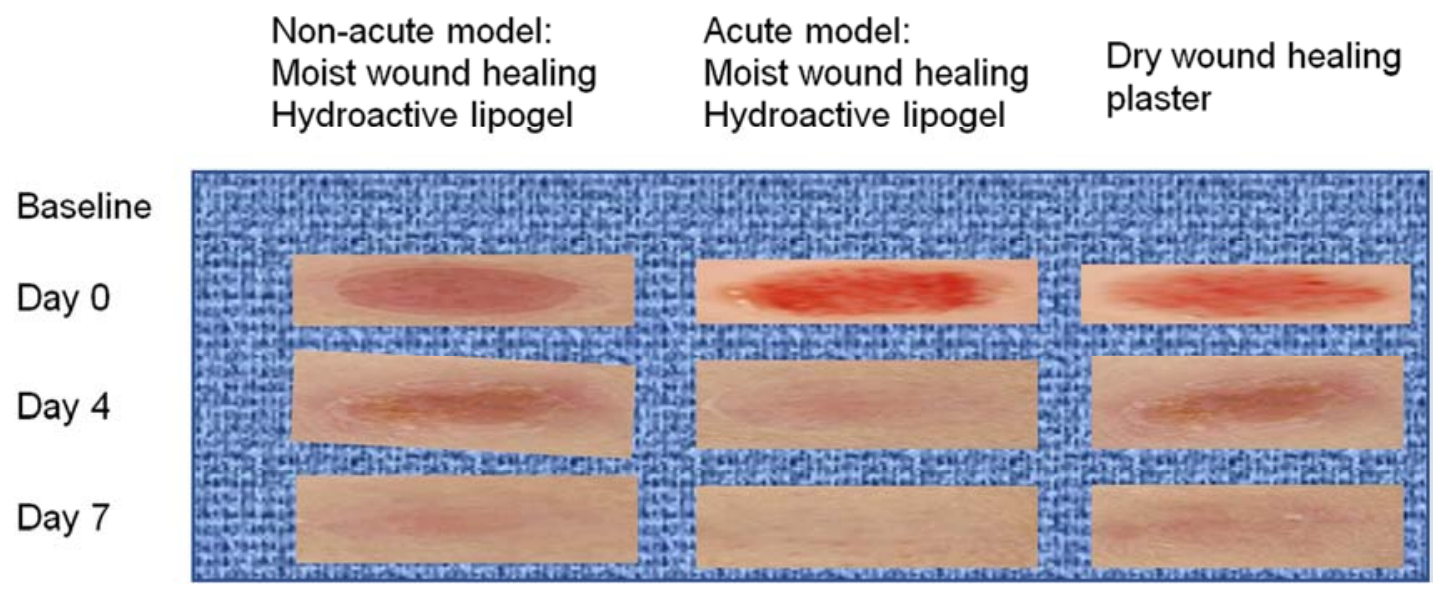

Figure 3. Representative video microscope images, documenting the healing progress of the three standardized lesions of patients at day 0 (baseline), day 3 and day 7 treated with the investigational product versus the reference standard plaster in acute (5 \pm 1 hour old incision) versus non-acute (14 \pm 2 days old incision) model.

\subsubsection{Assessment of Safety and Local Effects}

Signs of inflammation/infection were assessed at each visit regarding to erythema, pain, odor, non-healing, excessive exudation and hyperthermia. Potential signs for allergic reactions were documented as well (erythema, edema and itching). Signs of inflammation/infection and/or signs of allergic skin reactions with a score $>0$ were reported as adverse events (AEs) irrespective of the treatment procedure (investigational product vs. standard plaster). Additionally, AEs and changes in concomitant medication were recorded.

In case of strong signs for allergic reaction or inflammation/infection, the intervention has been stopped. Last observation has to be carried forward for analysis and follow-up for safety reasons as long as the reaction persists (safety population SP).

\subsection{Objectives}

The primary objective was the comparison of the investigational product versus traditional treatment (standard plaster) relating to the wound area assessed by quantitative image analysis at day 3 and day 7, quantified as area under the curve (AUC) and relative differences to baseline over time. The statistical analysis has been done on the AUC values and was based on the FAS (Full Analysis Set). FAS included all subjects who did not violate any inclusion or exclusion criteria at the beginning of the trial, who received at least one application of the test products and for whom at least one post-baseline assessment was performed. Since the strict practice of the ITT (Intention To Treat) principle in clinical trials may lead to arguable situations, FAS was chosen according to the statistical principles for clinical trials ICH-GCP E9 to be able to exclude subjects from analysis in justified cases.

Safety parameters were documented and analyzed based on the SP (Safety Population; all subjects who received at least one application).

\subsection{Statistics}

For sample size estimation, a medium effect size of 0.75 was assumed for the investigational product and conventional treatment for wound healing according to previous studies $[8 ; 19]$. To assure a significant result for a two-sided $\alpha$ of 0.05 with a statistical power of $80 \%$, a sample size of $n=20$ was needed by using the nQuery software (version 5.0, Statsols, Boston, USA). To account for an assumed drop-out rate of about $10 \%$, a total size of $\mathrm{n}=22$ per group was calculated. The confirmatory analysis of the primary and secondary objective was planned as Wilcoxon signed-ranks test.

Differences regarding AE rate and in particular frequencies of inflammations/infections or allergic reactions between both study arms were evaluated and compared by Chisquared tests.

Statistical analysis was performed using SAS $^{\circledR}$ software for windows version 9.3 (SAS Institute, Cary, USA) and R software applying the R-package of Bornkamp, Pinheiro and Bretz using a significance level of $p=0.05$. Values given in 
the text and tables are means and related standard deviations unless otherwise stated.

\subsection{Quality Assurance}

Monitoring and source data verification (SDV) and an independent quality assurance unit auditing data integrity assured accuracy of reported trial data, compliance with the current approved protocol, Good Clinical Practice (GCP) and applicable regulations.

\section{Results}

\subsection{Study Population}

Overall, 69 subjects were enrolled and screened for this study and 65 of these subjects were randomized (Figure 4). All subjects were treated and evaluated according to the protocol and were analyzed as PP $(n=59)$, FAS population $(\mathrm{n}=61)$ and as SP $(\mathrm{n}=61)$.

For the acute wound model, 21 patients were enrolled, and 44 patients for the non-acute wound model.

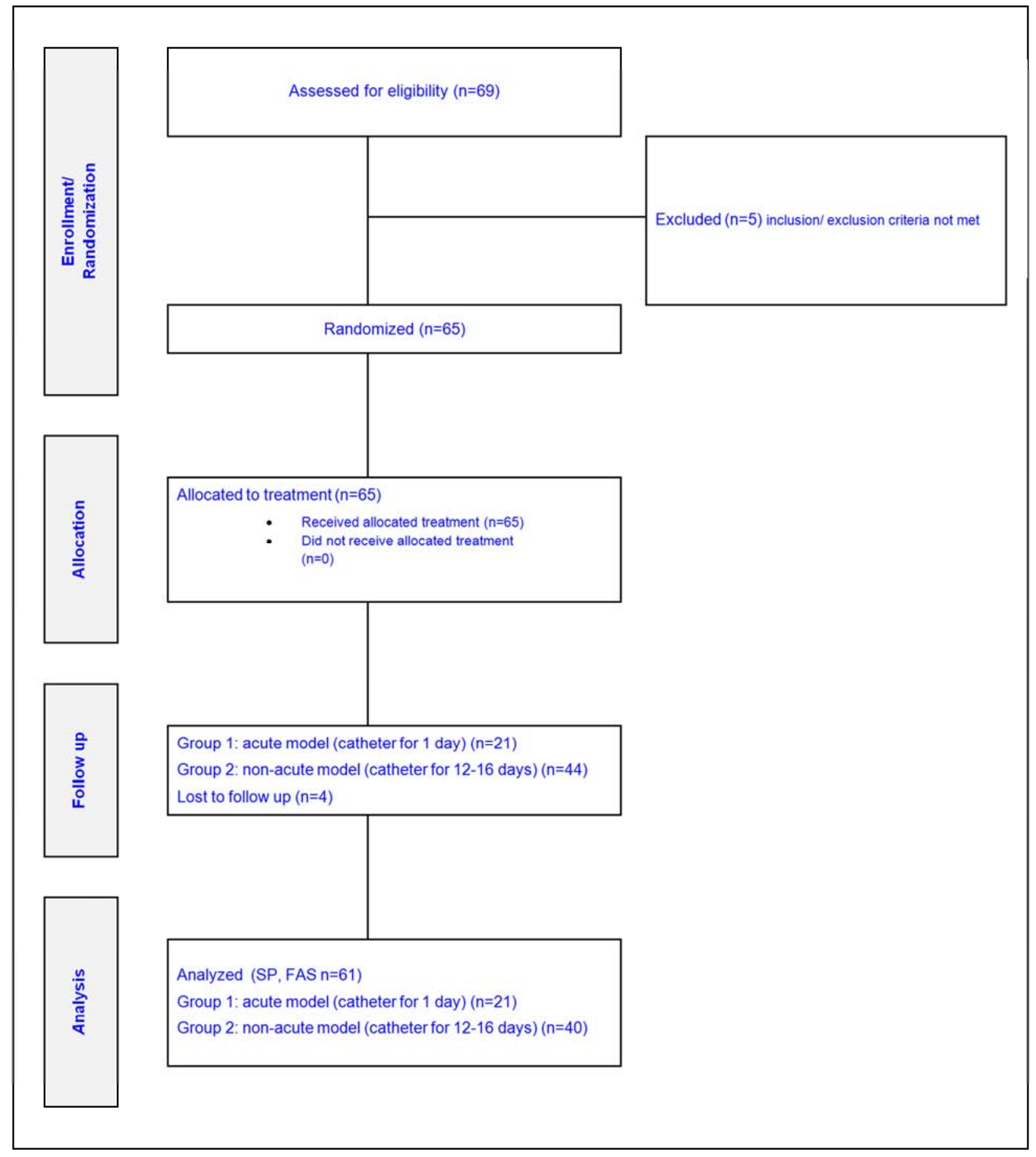

Figure 4. Disposition of subjects, separated into two groups representing acute and non-acute model. 


\subsection{Primary Objective: Comparison of Investigational Product vs. Traditional Treatment}

The confirmatory analysis was performed with the Wilcoxon signed-ranks test. The primary objective showed a significant reduction of wound areas treated with the investigational product. For non-acute wounds, a reduciton of $3319 \pm 5 * 1000 \mu \mathrm{m}^{2}$ (from $4123 \pm 182 * 1000 \mu \mathrm{m}^{2}$ to $804 \pm 162$ $\left.1000 \mu \mathrm{m}^{2}\right)$ has been detected in comparison to the conventionally treated incisions with a reduction of $2145 \pm 119$ $* 1000 \mu \mathrm{m}^{2}$ (from $3989 \pm 213$ to $1844 \pm 94 * 1000 \mu \mathrm{m}^{2}$ ) resulting in a significant difference at day $7(\mathrm{p}<0.001)$. For acute wounds, the reduction was $2145 \pm 138 * 1000 \mu \mathrm{m}^{2}$ versus $1713 \pm 94 * 1000 \mu \mathrm{m}^{2}$, respectively, $(\mathrm{p}<0.001)$. Faster wound healing was seen after application of the investigational product and the hypothesis of superiority versus traditional treatment with standard plaster could be accepted (Figure 5).

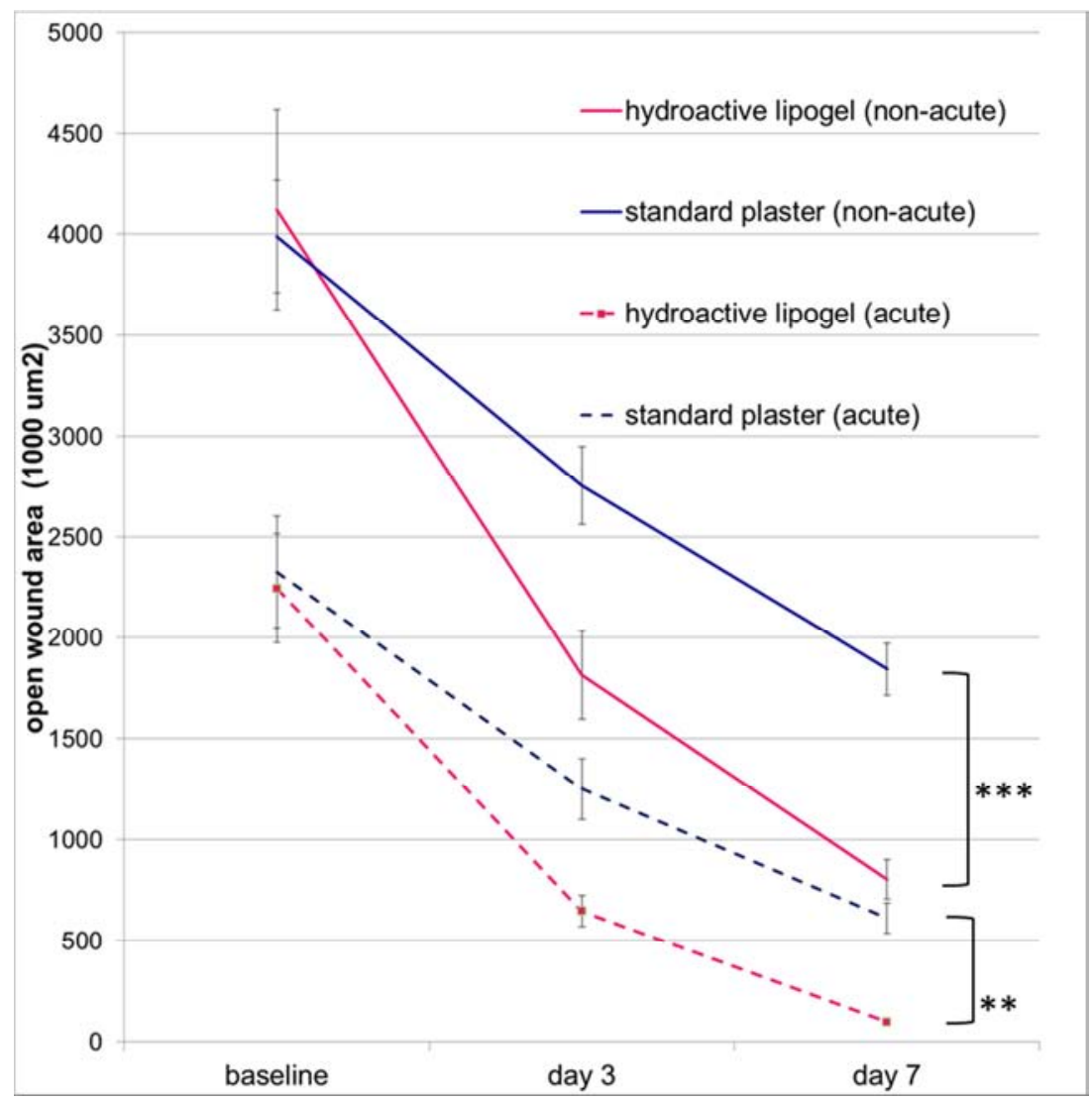

Figure 5. Line graph of mean open wound area by treatment against time for raw data. All assessment time points were significantly different for product (hydroactive lipogel, in pink) vs. traditional treatment (standard plaster, in blue) in both wound types (acute $=4-6$ hours, dashed lines) ( $p<0.01$ ) and non-acute $(14 \pm 2$ days, solid lines $)(p<0.001)$

\subsection{Secondary Objectives}

The relative differences to baseline for open wound area values by treatment over time are demonstrated in Figure 6 . Wound healing with standard plaster followed an almost linear course while healing at the areas treated with the investigational product was accelerated.

In the acute model, at day 7 the wounds treated by the hydroactive lipogel were almost closed with a remaining wound area of $98 \mathrm{um}^{2} \pm 58 * 1000 \mu \mathrm{m}^{2}$ (quantitative image analysis), while the remaining wound area under dry conditions was markedly larger $\left(614 \pm 77 * 1000 \mu \mathrm{m}^{2}\right)$. At day 3 , the hydroactive lipogel showed a closure rate of $71 \%$ versus $46 \%$ for plaster, and at day $7,95 \%$ versus $74 \%$, respectively, translating into a $30 \%$ improvement by lipogel $(\mathrm{p}<0.01)$.

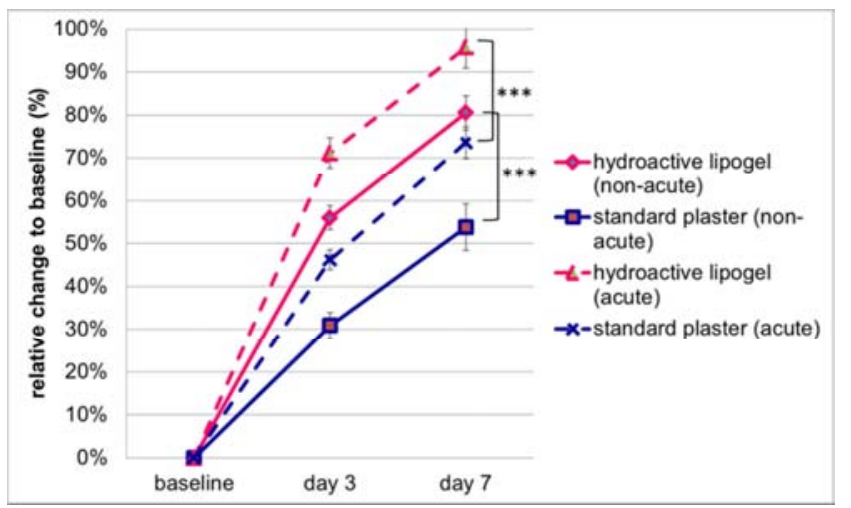

Figure 6. Line graph of mean open wound area/crusts by treatment against time for relative difference to baseline. All assessment time points were significantly different for product (hydroactive lipogel, in pink) vs. traditional treatment (standard plaster, in blue) in both wound types (acute $=4-6$ hours, dashed lines) $(p<0.001)$ and non-acute $(14 \pm 2$ days, solid lines $)(p<0.001)$. 
In the non-acute group, at day 7 the wounds treated with the investigational product presented a remaining wound area of $804 \pm 162 * 1000 \mu \mathrm{m}^{2}$ (quantitative image analysis), while the wounds in the (conventionally) comperator group were markedly larger with $1844 \mathrm{um}^{2} \pm 94 * 1000 \mu \mathrm{m}^{2}(\mathrm{p}<0.001)$. Hydroactive lipogel had a closure rate of $56 \%$ versus plaster (31\%), and at day $7,80.5 \%$ versus $54 \%$, respectively, translating into a $50 \%$ improvement with lipogel $(p<0.001)$.

\subsection{Safety Parameters}

A total of 7 AEs occurred in 7 subjects, 4 in the control arm, 3 in the lipogel group; all of them in the non-acute population. All events have been classified as non-serious AEs such as physiological signs of inflammation in relation to the mechanical wound induction by incision. All these events were observed at day 3 and no termination of application was necessary.

\section{Discussion}

The aim of this clinical study was to compare the wound healing properties of a lipogel promoting moist wound environment versus traditional treatment (standard plaster promoting a clean and dry environment) in a novel real-life wound model which affects all layers of the skin and suitable for inter-individual comparisons both in acute and non-acute wounds.

Test product performed better in promoting wound healing compared to traditional treatment as assessed by visual inspection and measured by quantitative image analysis and significantly shortened the treatment time for complete healing.

The modern wound healing product led to an exponential increase of wound closure, while the control group performing traditional treatment under dry conditions resulted in an almost linear progress in wound closure (Figure 5). This fact is indicating a prolonged inflammation phase and a reduced speed in re-epithelization and reparative processes for traditional wound treatment and thus confirming moist wound healing as "state of the art" being superior to dry wound healing.

This finding confirms previously reported results for an established abrasive wound model [8]. In addition, in a controlled study in animals [7], investigational product showed a significant advantage and faster healing properties in comparison to ointment saving 3 days for reepithelialization and closure of abrasive wounds [7]. Additionally, wound contraction supported by the function of myofibroblasts [9] was observed and allows to evaluate effects in the structures of the upper dermis (Stratum papillare) which revealed that moist environment reduces wound contraction [10].

For the group of non-acute wounds in our study, at day 7 the wounds treated with the investigational product were almost completely closed compared to the reference group which not yet had been closed (demonstrated by quantitative image analysis).
As demonstrated by the animal model [7], the treatment by lipogel is able to demonstrate an additional cosmetic benefit by reduction of scar formation.

In conclusion, wound healing was facilitated by moist environment provided by investigational product (hydroactive lipogel) both in abrasive and incisive wound models as well as in acute and non-acute wounds.

With regards to safety parameters (AE rates as well as frequencies of inflammation or allergic reaction), no significant differences between the two treatment options has been demonstrated. Wound induction by incision affects all layers of skin, physiological inflammation had to be expected at the beginning of the treatment course.

\section{Limitations}

Established wound models offer multiple advantages in assessing wound healing properties by different approaches. Such models allow standardized skin lesions which are comparable in size, surface area and wound depth. these techniques can generate standardized wounds of nearly identical size accurately reproduced by the same mode of action by same conditions enabling standardized comparisons and allowing representative conclusions in a limited number of patients.

Such categories of models are

1. Abrasive, affecting non-vascularized epidermis and opening capillary loops of dermal papillae leading to punctual bleeding [10], or

2. Non-invasive, such as measuring transepidermal water loss measurements (TEWL) in combination with tape stripping or suction blister wounds [17, 21, 21], or

3. Invasive, involving first-degree burns, laser, abrasion by using brushes or sandpaper and partial-thickness excisions or microdermabrasion by using a dermatome or

4. Invasive, such as mini-incisions, biopsy (punch or scalpel) affecting epidermis and dermis involving [11, 22].

In these models, physiological wound healing conditions are considered comparable for acute wounds without a special reference to the wounding mechanism (such as cuts, lacerations or superficial wounds) and results are considered assignable [11]. Therefore, it has to be pointed on the fact that, according to the affected tissues, results can differ in between the different models. In consequence, our model fits to category 4 with minor incisions through all three layers of the skin in a routine clinical procedure.

\section{Conclusion}

A moist wound environment is regarded superior and more conductive to healing than dry wound environment [1-4]. In our study, suggesting a novel real-life model of incisive wounds, we were able to confirm these findings, not only for acute but also non-acute wounds having progressed in the remodeling phase of wound repair: the investigational product demonstrated superior rates of re-epithelialization and superior 
cosmetic outcomes compared to traditional treatment (standard plaster) providing dry environment. In light of studies performed, moist environment provided by investigational product facilitates wound healing irrespective of the wound model (abrasive, incisive) and age of wound (acute, non-acute).

\section{References}

[1] National Health System - Northamptonshire Healthcare (2017). Guidelines for the assessment management of wounds. Retrieved from https://www.nhft.nhs.uk/download.cfm?doc=docm93jijm4n17 93.

[2] Australian Research Society (2019). Wound care evidence based guidelines summary. Retrieved from https://www.research.qut.au.html.

[3] Deutsche Gesellschaft für Kinderchirurgie e. V. (2013). AWMF Leitlinien-Register 006-129 Leitlinie zu Wunden und Wundbehandlung. Retrieved from https://www.awmf.org/leitlinien/detail/11/006-129.html.

[4] Deutsche Gesellschaft für Wundheilung und Wundbehandlung e. V. (2012). AWMF Leitlinien-Register 091/001S3-Leitlinie Lokaltherapie chronischer Wunden bei den Risiken CVI, PAVK und Diabetes mellitus. Retrieved from http://www.awmf.org/leitlinien/detail/11/091-001.html.

[5] Heyer K, Augustin M, Protz K, Herberger K, Spehr C, Rustenach SJ (2013). Effectiveness of advanced versus conventional wound dressings on healing of chronic wounds: systematic review and meta-analysis. Dermatology. 226 (2): 172-84. Retrieved from https://www.ncbi.nlm.nih.gov/pubmed/23711429.

[6] MediGel®. Information for Use (July 2016). Retrieved from www.medigel.de.

[7] Davis S, Gil J, Valdes J, Eberlein T (2013). Evaluating the efficacy of various over the counter topical formulations for stimulating epithelialization in a porcine partial thickness wound model. EWMA Kopenhagen Nov. 2013.

[8] Eberlein T, Gerke P, Lorenz H, Ammer R (2016). Advantages in wound healing by a topical easy to use wound healing lipogel for abrasive wounds-Evidence from a randomized, controlled experimental clinical study. Wound Medicine. Retrieved from https://doi.org/10.1016/j.wndm.2016.09.003.

[9] Martin P, Nunan R (2015). Cellular and molecular mechanisms of repair in acute and chronic wound healing. $\mathrm{Br}$ J Dermatol. 173 (2): 370-378. Retrieved from https://www.ncbi.nlm.nih.gov/pmc/articles/PMC4671308/.

[10] Wigger-Alberti W, Kuhlmann M, Ekanayake S, Wilhelm D (2009) Using a novel wound model to investigate the healing properties of products of superficial wounds. J Wound Care 18 (3): 123-128. Retrieved from https://www.ncbi.nlm.nih.gov/pubmed/19247233.

[11] Gottrup, F, Agren, MS and Karlsmark, T (2000). Models for use in wound healing research: a survey focusing on in vitro and in vivo adult soft tissue. Wound Repair Regen 8: 83-96. Retrieved https://www.ncbi.nlm.nih.gov/pubmed/10810034.

[12] Wilhelm K-P, Wilhelm D, Bielfeldt S (2016) Models of wound healing: an emphasis on clinical studies. Skin Res Technol. doi: 10.1111/srt.12317. Retrieved from https://www.ncbi.nlm.nih.gov/pubmed/27503009.

[13] Seldinger S (1953). Catheter replacement of the needle in percutaneous arteriography; a new technique. Acta radiol. 39 (5): 368-76. Retrieved from https://www.ncbi.nlm.nih.gov/pubmed/13057644.

[14] Conz PA, Dissegna D, Rodighiero MP, La Greca G (1997). Cannulation of the internal jugular vein: comparison of the classic Seldinger technique and an ultrasound guided method. J Nephrol. 10 (6): 311-3. Retrieved from https://www.ncbi.nlm.nih.gov/pubmed/9442443.

[15] Eming SA, Martin P, Tomic-Canic M (2014). Wound repair and regeneration: Mechanisms, signaling, and translation. Science translational medicine. 6 (265): 265. Retrieved from https://www.ncbi.nlm.nih.gov/pmc/articles/PMC4973620/.

[16] EUnetHTA (2013). Retrieved from https://www.eunethta.eu/methodological-guideline-for-rea-ofpharmaceuticals-direct-and-indirect-comparison/.

[17] Frodin T, Skogh M (1984). Measurement of transdermal water loss using an evaporimeter to follow the restitution of the barrier layer of human epidermis after stripping the stratum corneum. Acta Derm Venereol 64: 537-40. Retrieved from https://www.ncbi.nlm.nih.gov/pubmed/6084928.

[18] Fitzpatrick Classification of Skin Types I through VI. Published in: Fitzpatrick T (1988). The Validity and Practicality of Sun-Reactive Skin Types I Through VI. Arch Dermatol. 124 (6): 869-871. Retrieved from https://jamanetwork.com/journals/jamadermatology/articleabstract/549509.

[19] Descôteaux J (2007). Statistical power: An historical introduction. Tutorials in Quantitative Methods for Psychology. 3 (2): 28-34.

[20] Larsen HF, Ahlström MG, Gjerdrum LMR, Mogensen M, Ghathian K, Calum H, Sørensen AL, Forman JL, Vandeven M, Holerca MN, Du-Thumm L, Jorgensen LN, Ågren MS (2017). Noninvasive measurement of reepithelialization and microvascularity of suction-blister wounds with benchmarking to histology. Wound Repair Regeneration. 25 (6): 984-993. Retrieved https://www.ncbi.nlm.nih.gov/pubmed/29316016.

[21] Ahlström MG, Gjerdrum LMR, Larsen HF, Fuchs C, Sørensen AL, Forman JL, Ågren MS, Mogensen M (2018). Suction blister lesions and epithelialization monitored by optical coherence tomography. Skin Research and Technology. 24 (1): 65-72. Retrieved from https://www.ncbi.nlm.nih.gov/pubmed/28685861.

[22] Wilhelm KP, Wilhelm D, Bielfeldt S (2017). Models of wound healing: an emphasis on clinical studies. Skin Research and Technology. 23 (1): 3-12. Retrieved from https://www.ncbi.nlm.nih.gov/pubmed/27503009. 